\title{
International Linear Collider Global and Local Implications
}

\author{
Ryszard S. Romaniuk
}

\begin{abstract}
ILC machine - International Liner Collider, is one of two accelerators $\mathbf{e}+\mathbf{e}-$ just under design and advanced consideration to be built with final energy of colliding electron and positron beams over $1 \mathrm{TeV}$. An alternative project to ILC is CLIC in CERN The ILC machine is an important complementary addition for the research potential of the LHC accelerator complex. The required length of ILC is minimally $30 \mathrm{~km}$, but some versions of the TDR estimates mention nearly $50 \mathrm{~km}$. Superconducting RF linacs will be built using well established 1,3 GHz TESLA technology using ultrapure niobium or Nb3Sn resonant microwave cavities of $R R R$ class, of ultimate finesse, working with gradients over $35 \mathrm{MV} / \mathrm{m}$, while some versions of the design mention ultimate confinement as high as $50 \mathrm{MV} / \mathrm{m}$. Several teams from Poland (Kraków. Warszawa, Wroclaw - IFJ-PAN, AGH, UJ, NCBJ, UW, PW, PWr, INT-PAN) participate in the global design effort for this machine - including detectors, cryogenics, and SRF systems. Now it seems that the ILC machine will be built in Japan, during the period of 2016-2026. If true, Japan will turn to a world super-power in accelerator technology no.3 after CERN and USA. The paper summarizes the state-ofthe-art of technical and administration activities around the immense ILC and CLIC machines, with emphasis on potential participation of Polish teams in the global effort of newly established LCC - The Linear Collider Consortium.
\end{abstract}

Keywords-ILC, ILC-GDE, LCC, SRF, accelerators, particle colliders, linacs, superconductivity, high energy physics experiments, elementary particles, hadrons, electron beams, positron beams, microwave resonant cavities, high power $1,3 \mathrm{GHz}$ systems, superconducting RF electronics

\section{INTRODUCTION}

$\mathbf{N}$ OW, the longest linear accelerator for electrons is $3 \mathrm{~km}$ long machine in Stanford Laboratory (SLAC) of energy $50 \mathrm{GeV}$. Part of SLAC machine is used for powering the FEL laser - the LCLS. The ILC machine - International Linear Collider [1] is one of the most advanced designs of a double linear accelerator and $\mathrm{e}^{+} \mathrm{e}^{-}$collider, of final collision energy 1 TeV. Recently a key TDR document was released internationally for this machine, with co-authors from this country [2]. An alternative project to ILC is CERN's CLIC machine of target energy $3 \mathrm{TeV}$. The machine is expected to be an important addition for the research potential of the CERN's LHC accelerator complex of $14 \mathrm{TeV}$ proton collision energy, and its future upgrades. The required length of the warm CLIC machine (12 GHz working beam and $1 \mathrm{GHz}$ powering beam) is at least $30 \mathrm{~km}$, and some versions list 50 $\mathrm{km}$. Superconducting ILC linacs will be done in TESLA $1,3 \mathrm{GHz}$ technology, using microwave resonant cavities made

The author is with Warsaw University of Technology, Poland (e-mail: rrom@ise.pw.edu.pl) of pure niobium of RRR class or of $\mathrm{Nb}_{3} \mathrm{Sn}$, of high quality factor over $10^{11}$, working with the gradient over $35 \mathrm{MV} / \mathrm{m}$, and some designs list $50 \mathrm{MV} / \mathrm{m}$. Research and technical teams from Poland: Kraków, Warsaw, Wrocław - IFJ-PAN, AGH, UJ, NCBJ, UW, PW, PWr, INT-PAN, participate in the machine design, accelerator, detectors, cryogenics and control and measurement systems. Now, it is nearly decided that the machine is going to be built in Japan during the decade 20162026. If this happens, Japan turns to the third accelerator power in the world. In Germany, in DESY Hamburg, there is built a superconducting electron linac, $3 \mathrm{~km}$ in length, which will power the European XFEL laser. The control and fine timing system, as well as cryosystem is build with the participation of University teams from Poland (Warsaw, Wrocław and Łódź). The experiences gained by Polish teams during the design and construction of such machines as Zeus/HERA, CMS, TESLA, FLASH, EXFEL, JET, and others [3-17] will be effectively used to machines an order of magnitude bigger like ITER and ILC. Polish teams have recently also participated in large European projects on accelerator technology and infrastructures, what adds a lot to the ability to be accepted by and participate in global efforts [18-24].

The lack of large research international infrastructure in this country, of the European scale and of large discovery potential leaves the local communities out of the owners club. The large infrastructures are now ruling in particle physics, astronomy, chemistry, biology and medicine, environment protection and industry. Usually, such collaborations accept countries or institutions - owners of large domestic research infrastructures, who make them accessible for common usage in the European Union scale. It is called transnational access and is legally anchored in the EU FPs. National research communities, and in particular young researchers, increasingly frequently participate in large scientific and development experiments localized in Europe and globally in Japan, South Corea, China, South and North Americas. Such experiments have a common strength to ignite the imagination of young researchers. National experts from this country of different specialties of accelerator and laser technology are working in many European and global research centers like: APL, SOLEIL, ALBA, DESY, MAX-LAB, CEA, INFN, CERN, PSI, JLab, SLAC, J-PARC, KEK, and others. Gathering them together gives enough expertise to build in this country a large accelerator center, such as POLFEL or early IFMIF in Świerk National Center for Nuclear Research or in IFJ in Kraków. One of the solid planned infrastructures which will be built soon and attracts a number of experts from this country is the ILC. 
This paper was prepared using public and Intranet materials from the ILC, CLIC, LCC, TIARA and EuCARD web pages and work packages of these projects. The paper was presented during the 2013 WILGA Symposium on Electronics for HEP Experiments [3], and was published in Polish version in [25].

\section{LINEAR COLLIDER CONSORTIUM - LCC}

In February 2013 two analogous, but technologically different projects: cold ILC and warm CLIC created a common coordination body - The Linear Collider Collaboration - LCC. CLIC and ILC are two the most advanced research projects of the future experimental particle physics. The Consortium was created in order to supplement the world largest circular accelerator experiment now - the LHC. Why linacs, and not the next circular machine? Of course the next circular machines are also planned. The SLHC of the circle length 80 or $100 \mathrm{~km}$ is planed behind the 2035. Circular accelerator efficiently works with heavier particles like hadrons. For leptons, the basic confinement is synchrotron radiation. The LEP accelerator, predecessor of the LHC, in the same tunnel, was confined because of synchrotron radiation up to $210 \mathrm{GeV}$. Synchrotron radiation is inversely proportional to the fourth power of the mass of accelerated particles. Despite the energy of lepton collisions ( $1 \mathrm{TeV})$ in ILC is smaller than hadrons collisions in LHC (14 TeV), the measurements in ILC my be done with much bigger accuracy. The collisions between electrons and positrons are much simpler than the collisions of complex hadrons, and actually the collisions of included in them quarks, antiquarks and gluons, which are elementary constituents of baryonic matter. The collision energy of hadrons is distributed non homogeneously on partons and the image of collisions is very complex. The basic role of ILC in this situation is making of precision measurements of particles discovered by LHC, including Higgs boson. The measured quantities are: potential measurement of masses, numbers, dimensions, shapes, $\mathrm{TeV}$, additional dimensions, of light super-symmetric particles - possible candidates for dark matter, potential sources of dark energy - which is a total dominant, if it goes of the universe filling.

Establishment of the Linear Collider Consortium LCC is a big event, nearly without a precedence. Establishment of LCC increases greatly the chances for a fast building of a giant linear collider of really global scale, near to the scale of the LHC - the biggest machine ever. Much closer to the realization is ILC infrastructure, particularly, after the clear declaration of Japanese government to finance half of the machine costs, now estimated conservatively for 25 mld US\$. CERN's CLIC presents, however, a very attractive parameters and research potential. In CERN, the financial dominant is now decidedly the LHC, and will remain so during the next decade. The finances are not available for all projects, thus, the Japanese initiative to co-finance strongly the massive project of ILC is more than welcome by the world accelerator community. Actually, this valuable initiative was received with great joy and hope in a global scale.

\section{COMPACT LINEAR COLLIDER - CLIC}

CLIC - The Compact $\mathrm{e}^{+} \mathrm{e}^{-}$Linear Collider is a design of a machine of the collision energy of electrons and positrons over $3 \mathrm{TeV}$ and expandable to over $10 \mathrm{TeV}$, at the total length of both linacs over $50 \mathrm{~km}$. The project is based on an innovative conception of particle acceleration by a second initially accelerated and next decelerated particle beam of large intensity. Conventional solution of a collider of this energy would require several tens of thousands of high power klystrons, and machine length well over $100 \mathrm{~km}$. Compact solution, with powering second beam shortens this length by two to $50 \mathrm{~km}$, and reduces the number of klystrons to approximately 2000. In the two beam solution, the beams are propagated in parallel but in opposite directions. Colliding beams, of 1,5 TeV energy each, of $12 \mathrm{GHz}$ RF field frequency, and $100 \mathrm{MV} / \mathrm{m}$ gradient collide in the detector half length of the machine, summing the collision energy to $3 \mathrm{TeV}$. Powering beams serve as energy source for the colliding beams. Powering beams run in parallel to the colliding beams but begin in the machine center (where the collision point and detector is located for colliding beams). Maximal energy of powering beams is $2,4 \mathrm{GeV}$, and frequency is $1 \mathrm{GHz}$, excited by 2000 klystrons. This maximum energy is obtained at both ends of the machine. Powering beam is returned and divided to 24 sectors of the accelerated beam. The pulsed powering beam of high current $100 \mathrm{~A}$, is finally a source of short, lasting 240 ns, and very strong, high power RF pulses. The powering beam is decelerated from $2,4 \mathrm{GeV}$ to $240 \mathrm{MeV}$. The bunches of both beams run in full phase coincidence. Perfect synchronization gives maximum power transfer.

Deceleration structure in the powering beam channel decelerates the beam, what is a source of synchrotron braking field, and what causes highly efficient energy transfer, even at the level of $95 \%$ from the decelerated beam to the accelerated beam. Energy of the main accelerated beam, of 1,2 A current intensity, is increased from $9 \mathrm{GeV}$ to $1,5 \mathrm{TeV}$ in the field of frequency $12 \mathrm{GHz}$ and power $68 \mathrm{MW}$. The path of the main beam is the same for electrons and positrons and consists of 3 $\mathrm{GeV}$ injector, cooling rings, first bunch compressor, $6 \mathrm{GeV}$ booster linac, transmission lines for electrons and positrons on the beginning of the main linacs, second compressor of electron and positron bunches, main linac, precise magnetic systems for carrying, stabilizing, and focusing both beam for the interaction point (IP), interaction point surrounded by a complex multilayer detector.

The powering beam channel consists of two identical branches for the main electron and positron linacs: $1 \mathrm{~km}$ accelerator of powering beam, powered by 326 klystrons of 33 MW power and pulse duration time 140 microsecond, output beam energy 2,4 GeV, field frequency $1 \mathrm{GHz}$, delay loops, accumulation rings, transport system of powering beam distributed along the main linac, 24 section decelerators each 1 $\mathrm{km}$ long, couplers of the powering field to the main linac, and section beam dumps for the decelerated powering beam.

The technical issues under research now are: efficient generation of the powering beam of very high current above $100 \mathrm{~A}$ and large density, building of efficient extraction and transfer structures of high power from the decelerated beam to 
the accelerated beam; building of the main linac consisting of warm $12 \mathrm{GHz}$ microwave resonant structures with travelling wave and field gradient over $100 \mathrm{MV} / \mathrm{m}$; research on the final collision energy after upgrades of the machine reaching over 5 $\mathrm{TeV}$; generation of the main beam of high energy and minimized emittance; keeping low emittance during beam acceleration and transport, beam focusing and stability issues in the interaction point - what requires sub-nanometer accuracy in beam positioning, what in turn requires submicrometer accuracy of positioning of many accelerator parts, close to the interaction point.

\section{ILC MACHINE AND ITS GLOBAL AND LOCAL IMPACT}

The International linear collider was born as a combination of several regional an global projects, into a really global one. The ideas were: Global Collider - GLC, Next Generation Collider NLC, and the most technologically advanced TESLA project - Teraelectronovolt Energy Superconducting Linear Accelerator proposed by DESY. The "Panel of Wise" was established in 2004 - ITRP (International Technology Recommendation Panel) to decide on the technology of choice. The choice was cold technology and a single machine - The ILC. Reference Design Report RDR was published in 2007, in big degree using the excellent, deep, multivolume TDR (Technical Design Report) document for TESLA. TESLA project was to be realized in German Synchrotron Center DESY in Hamburg, with strong international participation, including strong participation of Poland. In 2005, the International Committee for Future Accelerators ICFA called an international body Global Design Effort GDE to run initial stages of the ILC project. Now the functions of GDE were undertaken by the LCC Collaboration. Strengthening of the motivation to build the ILC was the discovery of the $125 \mathrm{GeV}$ Higgs boson. ILC offers precise measurements of properties of the newly discovered boson during the work in the area of Higgs field radiation between 115 and $250 \mathrm{GeV}$. Apart of Higgs field research, the ILC will have the possibility to work in the area of energies $350 \mathrm{GeV}$, or in the vicinity of the top quark. It is expected that vacuum stability may also be measured. Actually, the ILC is a real Higgs factory.

The accelerating process starts with the source of charged particles. The ILC is an electron - positron accelerator. The electron gun in ILC will be cold. It bases on electron photoemission from a photocathode. A cloud of electrons is emitted from photocathode by means of nanosecond UV laser pulse. Such a method gives electron cloud highly polarized. Electron cloud is focused initially into a bunch and next a beam of initially accelerated stream of bunches in the first part of the machine of the length several hundred $\mathrm{m}$ is directed to the further parts of the machine towards the main linac. This part of the machine is called an injector. The output beam from the injector has $5 \mathrm{GeV}$ energy. In this place, there is produced positron beam from the electron beam. Electron beam strikes a thin metal target from the titanium alloy, where it is strongly decelerated. The synchrotron radiation from decelerated, highly energetic electron beam produces highly polarized pairs electron - positron. The pairs are split and formed into two separate beams - electron and positron and next accelerated to equal energies of $5 \mathrm{GeV}$ in separate linacs. Forming of short 5 $\mathrm{GeV}$ bunches of electrons and positrons takes place in the cooling rings and next accumulation rings. Finally, the bunches are $6 \mathrm{~mm}$ in length, and the vertical and horizontal emittances are respectively: $2 \mathrm{pm}$ and $600 \mathrm{pm}$. The bunches are transported from the cooling rings to two main superconducting linacs, where are accelerated to the energy $350 \mathrm{GeV}$, each on the length of $11 \mathrm{~km}$. The power of each beam is over $5 \mathrm{MW}$. Work frequency of the linac is $5 \mathrm{~Hz}$. In the interaction region the beams are strongly focuses to the dimensions of several to several hundred nm, in order to increase the brilliance and next are subject to collision in one of two nondependent and complementary particle detectors ILD and SiD.

\section{ELECTRON - POSITRON ANNIHILATION $\mathrm{E}^{+}-\mathrm{E}^{-}$}

High energy collisions of protons lead to creation of many new products. Hadrons are objects of complex internal structure, which additionally depends on their energy. The electron - positron annihilation is completely different. An intermediate choice is lepton - hadron collision, where lepton is scattered on the internal structure of the hadron, sampling by penetration in this way its structure. Completely different field, are heavy ions collisions like $\mathrm{Pb}$, where a real fireball is created, or quark - gluon plasma QGP, from which during cooling escape particle jets. Electron - positron annihilation is characterized by energy cleanliness and low collision multiplicity. At low energies, the annihilation produces only X-ray photons. Proton - proton collision in the LHC leads on the average to a few tens of collisions during a single bunch crossing, taking place now every 50 ns, and finally every 25 ns. Each of these collisions, depending of its hardness, leads to generation of a few hundred of high energy particles. The image of such collisions is very complex, and reconstruction of this image starts from identification of all collision vertexes and identification of created particles and energy calculation, including the lacking energy. During the lepton collisions, in the ILC there is created a dense photon cloud, in which there are secondary photon-photon collisions. These collisions are the background of the event. It is estimated, that each bunch crossing in ILC will lead to at least single photon-photon collision. These collisions lead, in the final state, to production of several hadrons. Each $\mathrm{e}^{+}-\mathrm{e}^{-}$bunch crossing produces many secondary pairs of electron - positron pairs confined to a very small volume around the beam.

The difference in collision characteristics between hadrons (LHC) and leptons (ILC) have essential influence on the construction of detectors. The LHC detectors are of hybrid construction, multichannel, multilayer, radiation hardened, possess strong solenoids to enable particle differentiation. They have complex triggering systems, catching from the sea of events only the most interesting cases. Large muon detectors are outside the solenoid. In the ILC, the detector may be much smaller, more concentrated around the interaction point. The first layer of the pixel vertex detector is very close to the real vertex of each collision. The whole detector, from the vertex detector to calorimeters, may be positioned inside 
the solenoid. The resolution of measurement of momentum. From the measurements in trackers, may be increased over 10 times in comparison with the LHC. The energy of particle jets may be measured with the resolution several times better. Determination of quantum numbers of generated quarks is simpler. There are no existing accumulations, which are difficult for disentangling, and which saturate the LHC detectors, and complicate the measurement process. The collisions in the ILC are clean, with much less and more homogeneous multiplicity.

The elementary coupling of photon is the same for all kinds of elementary particles as quarks and leptons. It is also the same for other hypothetic particles outside the SM. Thus, the $\mathrm{e}^{+}-\mathrm{e}^{-}$annihilation, of sufficiently large energy produce pairs of all particles with equal frequency. It concerns also the new, unknown and exotic particles. One says, that the collisions in ILC are more 'democratic', than in LHC. For large energy, the main annihilation processes, according to the SM model are: annihilation to quark and lepton pairs, annihilation to $\mathrm{W}^{+} \mathrm{W}^{-}$, and production of single $\mathrm{W}$ and $\mathrm{Z}$. Production of new particles may be easily distinguishable from these processes, because of completely different cross sections. IP detectors do not require any data trigger, because of easy event differentiation. All bunch crossings are recorded and the event reduction is done offline. The ILC does not require classification selection of events, differently than in LHC. W and Z bosons are easily recognized by the kind of hadron decay. ILC produces Higgs boson more frequently and is more characteristic than in LHC. It is $1 \%$ of all $\mathrm{e}^{+} \mathrm{e}^{-}$annihilations. Typical reaction is $\mathrm{e}^{+} \mathrm{e}^{-}-\mathrm{ZH}$.

Linear accelerator is more scalable than circular one. It may work with different collision energy, differently than in circular accelerator, which works at the set value of energy. Maximal energy is defined by the accelerator length and number of active components on the beam path. Lowering of the energy is possible at the cost of beam brilliance proportional approximately to the reduced energy. In the case of essential observations in the energy maximum, it is possible to increase it further. This energy flexibility of linac enables experiment design as if it were done at different machines. For example: energy range $9-160 \mathrm{GeV}$ is equivalent to the $\mathrm{Z}$ resonance and threshold $\mathrm{e}^{+} \mathrm{e}^{-}--\mathrm{W}^{+} \mathrm{W}^{-} ; 250 \mathrm{GeV}$ is equivalent to the maximum cross section for $\mathrm{e}^{+} \mathrm{e}^{-}-\mathrm{ZH} ; 350-400 \mathrm{GeV}$ is compatible with the production of up quark; $500 \mathrm{GeV}$ is equivalent of fermionic reaction $\mathrm{e}^{+} \mathrm{e}^{-}-\mathrm{ff}^{-} ; 1000 \mathrm{GeV}$ is equivalent to Higgs boson coupling with up quark and self coupling.

\section{ILC IN POLAND}

Polish research groups active in accelerator technology are dissipated at various large accelerator infrastructures in Europe and elsewhere like Japan, JINR in Dubna, USA, Korea, etc. Many of these groups participated in the global effort of preparation of the TDR of ILC. Many declared further active participation in ILC design, construction and commissioning of its particular components. The expertise of Polish teams, relevant to the ILC design, is in the fields of cryogenics, TESLA cavities, SRF technology, LLRF technology, high power microwaves, beam dynamics, control systems, and experiment simulations.

\section{CONCLUSIONS}

Many arguments speak for building of a large and widely scalable positron - electron annihilation superconducting machine. Discovery of the Higgs boson considerably increases this demand. The planed infrastructure is perfectly complementary to the LHC. Life time of the LHC is predicted, in the current form, together with a few upgrades, till 2035. Construction of ILC will overlap with this period. If the construction starts in 2016 and will last for a decade, then the research will start in 2026. The best results the ILC will gather at the end of LHC life.

\section{ACKNOWLEDGMENTS}

The author would like to thank EuCARD management in CERN for extremely efficient cooperation.

\section{REFERENCES}

[1] International Linear Collider: www.linarcollider.org

[2] ILC Technical Design Report, CERN, FermiLab, KEK, 2013

[3] R.S.Romaniuk, Photonics applications and Web engineering: Wilga May 2013, Proc.SPIE 8903, art.no.890303, 2013

[4] R.S.Romaniuk, Accelerator technology and high energy physics experiments, Photonics Applications and Web Engineering, Wilga May 2012, Proc.SPIE, vol. 8454, art no. 845404, 2012.

[5] R.S. Romaniuk, Photon physics and plasma research, Photonics Applications and Web Engineering, Wilga May 2012, Proc.SPIE vol. 8454, art. no. 845405, 2012.

[6] R.S.Romaniuk, Wilga photonics and web engineering, January 2012, Proc.SPIE, vol. 8454, art no. 845402, 2012.

[7] R.Romaniuk, K.Pozniak, Metrological aspects of accelerator technology and high energy physics experiments, Measurement Science and Technology, vol.18. no.8, art.no.E01, 2008.

[8] T.Czarski, K.T.Pozniak, R.S.Romaniuk, et al., Superconducting cavity driving with fpga controller, Nuclear Instruments and Methods in Physics Research A, vol.568, no.2, pp.854-862, 2006.

[9] T.Czarski, K.T.Pozniak, R.S.Romaniuk, et al., TESLA cavity modeling and digital implementation in fpga technology for control system development, Nuclear Instruments and Methods in Physics Research A, vol.556, no.2, pp.565-576, 2006.

[10] T.Czarski, K.T.Pozniak, R.S.Romaniuk, et al., Cavity parameters identification for TESLA control system development, Nuclear Instruments and Methods in Physics Research A, vol.548, no.3, pp.283297, 2005.

[11] R.Romaniuk, Wilga photonics and web engineering 2010, Photonics Letters of Poland, vol.2, no.2, pp.55-57, 2010.

[12] R.Romaniuk, Wilga symposium on photonics applications, Photonic Letters of Poland, vol.1, no.2, pp.46-48, 2009.

[13] R.Romaniuk, Photonics and web engineering 2011, International Journal of Electronics and Telecommunications, vol.57, no.3, pp.421428, 2011.

[14] R.Romaniuk, Advanced photonic and electronic systems Wilga 2010, International Journal of Electronics and Telecommunications, vol.56, no.4, pp.479-484, 2010.

[15] S.Chatrchyan, K.T.Pozniak, R.S.Romaniuk, W.Zabolotny, et al., The CMS Collaboration, Commissioning of the CMS experiment and the cosmic run at four tesla, Journal of Instrumentation, vol.5, no.3, art.no.T03001, 2010. 
[16] S.Chatrchyan, K.T.Pozniak, R.S.Romaniuk, W.Zabolotny, et al., The CMS Collaboration, Performance of the CMS Level-1 trigger during commissioning with cosmic ray muons and LHC beams, Journal of Instrumentation vol.5, no.3, art.no.T03002, 2010.

[17] S.Chatrchyan, K.T.Pozniak, R.S.Romaniuk, W.Zabolotny, et al., The CMS Collaboration, Performance of the CMS drift-tube chamber local trigger with cosmic rays, Journal of Instrumentation, vol.5, no.3, art.no.T03003, 2010.

[18] R.S.Romaniuk, EuCARD2 - enhanced accelerator R\&D in Europe, Proc.SPIE 8903, art.no.89031P, 2013

[19] R.S.Romaniuk, Accelerators for society: succession of European infrastructural projects: CARE, EuCARD, TIARA and EuCARD2, Proc. SPIE 8903, art.no.890320, 2013

[20] R.S.Romaniuk, Visions of the future of particle accelerators, Proc.SPIE 8903, art.no. 890324, 2013
[21] R.S.Romaniuk, Accelerator science and technology in Europe 20082017, Proc.SPIE 8903, art.no.89031P

[22] R.S.Romaniuk, Accelerator science and technology in Europe EuCARD 2012, Proc.SPIE, vol. 8454, art no. 84540O, 2012.

[23] R.Romaniuk, Accelerator infrastructure in Europe EuCARD 2011, International Journal of Electronics and Telecommunications, vol.57, no.3, pp.413-419, 2011

[24] R.Romaniuk, EuCARD 2010 accelerator technology in Europe, International Journal of Electronics and Telecommunications, vol.56, no. 4, pp.485-488, 2010

[25] R.S.Romaniuk, Międzynarodowy zderzacz liniowy, Elektronika, vol. 54, no. 3, str.119-122 (2013) 